\title{
Patient-generated Digital Images after Pediatric Ambulatory Surgery
}

Matthew W. Miller'; Rachael K. Ross ${ }^{1}$; Christina Voight ${ }^{1}$; Heather Brouwer'; Dean J. Karavite ${ }^{3}$; Jeffrey S. Gerber1,2,4; Robert W. Grundmeier ${ }^{2,3}$; Susan E. Coffin ${ }^{1,2,4}$

'Division of Infectious Disease, Children's Hospital of Philadelphia, Philadelphia Pennsylvania;

2Department of Pediatrics, Perelman School of Medicine at the University of Pennsylvania, Philadelphia, Pennsylvania;

32Department of Biomedical and Health Informatics, Children's Hospital of Philadelphia, Philadelphia, Pennsylvania;

${ }^{4}$ Center of Pediatric Clinical Effectiveness, Children's Hospital of Philadelphia, Philadelphia, Pennsylvania

\section{Keywords}

Ambulatory care information systems, patient records, surgery, workflow, clinical images

\section{Summary}

Objective: To describe the use of digital images captured by parents or guardians and sent to clinicians for assessment of wounds after pediatric ambulatory surgery.

Methods: Subjects with digital images of post-operative wounds were identified as part of an ongoing cohort study of infections after ambulatory surgery within a large pediatric healthcare system. We performed a structured review of the electronic health record (EHR) to determine how digital images were documented in the EHR and used in clinical care.

Results: We identified 166 patients whose parent or guardian reported sending a digital image of the wound to the clinician after surgery. A corresponding digital image was located in the EHR in only 121 of these encounters. A change in clinical management was documented in $20 \%$ of these encounters, including referral for in-person evaluation of the wound and antibiotic prescription. Conclusion: Clinical teams have developed ad hoc workflows to use digital images to evaluate post-operative pediatric surgical patients. Because the use of digital images to support follow-up care after ambulatory surgery is likely to increase, it is important that high-quality images are captured and documented appropriately in the EHR to ensure privacy, security, and a high-level of care.

\section{Correspondence to}

Matthew Miller, BS

Clinical Research Assistant

The Children's Hospital of Philadelphia

3535 Market Street, Suite 1512

Philadelphia, PA 19104

Phone: 215.590 .3289

Email: millerm12@email.chop.edu
Appl Clin Inform 2016; 7: 646-652

http://dx.doi.org/10.4338/ACl-2015-12-CR-0168

received: December 1, 2015

accepted: May 4, 2016

published: July 6, 2016

Citation: Miller MW; Ross RK; Voight C; Brouwer H; Karavite DJ; Gerber JS; Grundmeier RW; Coffin SE. Patient-generated Digital Images after Pediatric Ambulatory Surgery. Appl Clin Inform 2016; 7: 646-652 http://dx.doi.org/10.4338/ACI-2015-12-CR-0168 


\section{Introduction}

The number of ambulatory surgeries performed annually in the United States continues to increase as a means to lower costs and improve quality of care [1]. A brief in-person visit with the surgeon is often conducted 1-2 weeks after the procedure to assess the effectiveness of the surgery and diagnose any complications [2]. Telehealth follow-up might provide an effective alternative to an in-person visit and reduce the burden for patients who must travel a significant distance, rearrange work schedules, or experience possible complications before a scheduled appointment. Prior studies have demonstrated that telehealth follow-up is an effective alternative to in-person visits, associated with reduced frequency of in-person follow-up visits while maintaining a satisfactory quality of care [3-5]. Remote follow-up has traditionally consisted of a telephone consultation, but with the ubiquity of mobile phones with digital cameras these encounters are increasingly supplemented with patient-generated digital images [6,7]. Clinical decisions based on the assessment of digital photos and related clinical information have been shown to be comparable to face-to-face evaluations in a range of specialties [8-10].

Some electronic health record (EHR) vendors have developed smart phone applications that allow providers to take clinical photos during the course of an office visit. However, EHR-linked applications that facilitate secure transmission, receipt, and upload of patient-generated images from personal mobile phones into the EHR are less frequently available [11]. As a result, some providers have developed their own informal practices to manage patient-captured images delivered to them through either email or text message. However, these informal practices may pose risks associated with potential loss of clinically relevant data and security breaches [12]. The goal of this case report is to describe how patient-captured digital images are integrated in the electronic health record and used in clinical decision-making within a pediatric care network.

\section{Methods}

\subsection{Study Design and Setting}

This cross-sectional study describes the use of patient-generated digital images by clinicians for the assessment of post-operative wounds. The study was conducted at The Children's Hospital of Philadelphia (CHOP), a pediatric healthcare network, which includes a 526-bed hospital, three ambulatory surgical facilities (ASF), and 14 specialty offices. Approximately 6,000 ambulatory surgeries are performed at CHOP each year. All practice sites in the network share a single EHR (Epic ${ }^{\oplus}$, Verona, WI).

\subsection{Study Population and Data Sources}

The study population was assembled from an ongoing cohort study examining the incidence and risk factors associated with surgical site infections after ambulatory surgical procedures between February 2013 and June 2014. This study targeted patients aged 0 to 17 years of age who had ambulatory surgery completed in an operating room resulting in class I or II wounds requiring incision and closure. Data collection for this parent study was conducted via a structured telephone interview with the parent or legal guardian of subjects 30 to 45 days after the surgical procedure. Data were entered in real-time into a structured case report form. Respondents were asked to report all of the subject's healthcare contacts, use of antibiotics, and concerns about the wound since the surgery date. A select number of interviews were recorded and audited for quality assurance purposes.

\subsection{Data Collection and Analysis}

The data used in this report were generated by electronically searching interview data for all references to a digital image, such as "picture", "image", "pic", and others. Respondents were not specifically asked about images. For encounters where a digital image was mentioned in the interview, we manually reviewed the EHR to determine if and how images of the surgical wound were captured, 
documented, and used in medical-decision making. When a patient had multiple episodes of image transmission, we treated each episode as an independent event referred to as an 'encounter'. For each encounter we determined that there was 'documentation' if the image was referenced in the physician note, attached as an "encounter-level document", or embedded (i.e. copied and pasted) in the note itself. This distinction was made to consistently categorize the location of each image within the EHR. We captured data on all images sent within 45-days of the surgery.

The data were collected and stored in REDCap, a secure, web-based application for building and managing online surveys and databases [13]. Data were summarized using frequencies and percentages for categorical variables, medians and interquartile ranges for continuous data.

\section{Results}

A parent mentioned sending a digital image in 166 (3\%) of the 5865 interviews ( $>$ Figure 1). Upon chart review, there was documentation of one or more digital images in the EHR for 121 of these 166 patients (73\%). Among these 121 patients, there were a total 163 individual encounters with EHR documentation of a digital image ( $>$ Figure 1$)$. Most patients $(88,73 \%)$ had a single encounter with documentation of a digital image during the 45-day period, although some patients had up to 4 encounters for which digital images were documented.

Among the 163 encounters with documentation of an image, 42 (26\%) did not include an image file in the EHR. Of the 121 (74\%) encounters with an image file, 96 (79\%) had images embedded in an encounter note and $25(21 \%)$ had images that were attached as an encounter-level document.

Urology $(n=101 ; 62 \%)$ and plastic surgery $(n=34 ; 21 \%)$ were the most common surgical specialties to have documentation of an image associated with an encounter ( $>$ Table 1). Additionally, these specialties had the highest rate of uploaded digital images in the EHR, $86 \%$ and $79 \%$ respectively.

- Table 2 describes the medical decisions made at encounters where a digital image was sent. For most encounters (79\%), there was no change in clinical management. An appointment with the surgeon's office was the most common clinical action taken (7\%) at these encounters.

\section{Discussion}

This study describes how patient-generated digital images were integrated into one institution's EHR and used for medical decision-making after ambulatory surgery. We found that most encounters with documentation of a digital image resulted in no clinical action. Additionally, we observed inconsistent documentation of these digital images in the EHR, raising concerns about potential loss of data, breeches in patient privacy, and storage location.

Prior studies have shown that use of remote follow-up after ambulatory surgery reduces unnecessary office visits and patient travel [3-5]. In our study, we found that changes in clinical management were relatively uncommon in encounters with a documented digital image. This suggests that the use of digital images may help avoid in-person post-operative visits that are unlikely to trigger a change in management.

As the use of digital images increases, it is important that they are uploaded and appropriately documented in the EHR. We found that there was a significant amount of clinically relevant information lost at each step of receiving, processing and documenting digital images. We recommend that all patient-generated digital images received by a clinician should be documented in the EHR with details about who sent the picture, when it was sent, and its role in the clinical decision-making process [14].

We found that most uploaded images were copied and then pasted into the encounter note as opposed to attached as a document. This process might interfere with future attempts to retrieve the image, because some EHRs do not catalogue the presence of an embedded image. Consequently, the encounter does not have a signifier indicating there is an associated image, the image is not findable by standard search mechanisms, and the image cannot be efficiently used outside the note where it was embedded (e.g. as an attachment to messages between clinicians). One study found that pro- 
viders prefer embedding clinical images, rather than attaching them, in encounter notes to ensure that all relevant patient information is kept in one location [11]. Providers might be more likely to attach an image if there were secure and standard EHR workflows to attach (rather than embed) patient-submitted photos, and if there were mechanisms to display the attached images alongside the related narrative documentation.

At the time of our study, there was no method for parents to send digital images securely to providers in our network, so images had to be sent via text or email. The systems used were unlikely to be secure for the transfer of patient information because the transmission was unencrypted and did not utilize the healthcare network's secure network $[11,12]$. Furthermore, after review of the image, there was no way to track if the photos were deleted or retained on these devices/accounts, which do not permit the secure storage of patient health information. As the use of digital images increases, healthcare networks need to establish processes that provide patients secure and efficient mechanisms to share digital images with their providers. Many vendors of EHRs are creating new features, such as patient portals, that facilitate sending photos to providers [11]. More research is needed to determine if the adoption of these features improves the current workflow and reduces data loss and debris.

Ascertainment bias is a significant limitation of this study. Specifically, we did not directly ask caregivers if they had sent an image of the surgical wound to a provider. Thus, we believe our data likely underestimated the frequency with which digital images were used by parents to describe their child's wound.

\section{Conclusion}

After ambulatory surgeries, when parents send pictures via email or text to providers for evaluation it is important that systems in place ensure the secure transfer of images and facilitate appropriate documentation in the EHR. This is necessary to avoid the loss of information and fully utilize images in patient care.

\section{Clinical Relevance Statement}

When parents send pictures to clinicians for evaluation after ambulatory surgery it is important that these images are securely transferred with appropriate documentation in the EHR.

\section{Conflict of Interest}

No author had any conflicts of interest regarding this study.

\section{Human Subjects Protections}

This study was approved by the Children's Hospital of Philadelphia institutional review board to ensure the protection of all human subjects. The review board's guidelines are in compliance with the World Medical Association Declaration of Helsinki on Ethical Principles for Medical Research Involving Human Subjects.

\section{Acknowledgments}

Brandon Putz and Cory Matchulat performed data collection for this study, which was crucial to the success of this report. 


\section{5}

Total interviews completed during study period

5699 (97\%)

No report of digital image sent by caretaker

\section{$166(3 \%)$}

Caretakers reported sending digital image to provider

\section{$45(27 \%)$}

Patients with no documentation of digital image in EHR

121 (73\%) Patients had 163

individual encounters with

documentation of a digital image

Fig. 1 Digital images reported after ambulatory surgery. 
Table 1 Documentation of digital images by surgical specialty

\begin{tabular}{|l|l|l|l|}
\hline & $\begin{array}{l}\text { Encounters with documentation in } \\
\text { EHR }\end{array}$ & \multicolumn{2}{|l|}{ Encounters with Image in EHR } \\
\cline { 3 - 4 } & 101 & $\mathbf{N}$ & $\%$ \\
\hline Urology & 34 & 87 & $86 \%$ \\
\hline Plastic Surgery & 13 & 27 & $78 \%$ \\
\hline Ophthalmology & 6 & 2 & $15 \%$ \\
\hline Otolaryngology & 5 & 2 & $33 \%$ \\
\hline Orthopedics & 4 & 1 & $20 \%$ \\
\hline General Surgery & 163 & 2 & $50 \%$ \\
\hline Total & & 121 & $74 \%$ \\
\hline
\end{tabular}

Table 2 Clinical actions taken after encounters where digital images were sent $(n=163)$. Encounters could have multiple clinical actions taken.

\begin{tabular}{|l|l|l|}
\hline \multirow{2}{*}{ Clinical Action } & \multicolumn{2}{|l|}{ Encounters with Clinical Actions } \\
\cline { 2 - 3 } & N & $\%$ \\
\hline No action taken & 134 & $78.4 \%$ \\
\hline Appt. with Surgeon's Office & 11 & $6.4 \%$ \\
\hline Suggested over-the-counter medication & 10 & $5.9 \%$ \\
\hline Prescribed antibiotic & 9 & $5.2 \%$ \\
\hline Prescribed other & 4 & $2.3 \%$ \\
\hline Appt. with primary care physician & 2 & $1.2 \%$ \\
\hline Sent to emergency room & 1 & $0.6 \%$ \\
\hline Total & 171 & $100 \%$ \\
\hline
\end{tabular}




\section{References}

1. Munnich EL, Parente ST. Procedures take less time at ambulatory surgery centers, keeping costs down and ability to meet demand up. Health Affair 2014; 33(5): 764-769.

2. Hwa K, Wren SM. Telehealth follow-up in lieu of postoperative clinic visit for ambulatory surgery: results of a pilot program. JAMA Surg 2013; 148(9): 823-827.

3. Wasson J, Gaudette C, Waley F, Sauvigne A, Baribeau P, Welch HG. Telephone Care as a Substitute for Routine Clinic Follow-up. JAMA 1992; 267(13): 1788-1793.

4. Kassmann BP, Docherty SL, Rice HE, Bailey DE, Schweitzer M. Telephone Follow-up for Pediatric Ambulatory Surgery: Parent and Provider Satisfaction. J Pediatr Nurs 2012; 27(6): 715-724.

5. McVay MR, Kelley KR, Mathews DL, Jackson RJ, Kokoska ER, Smith SD. Postoperative follow-up: is a phone call enough? J Pediatr Surg 2008; 43(1): 83-86.

6. Hsieh C-H, Tsai H-H, Yin J-W, Chen C-Y, Yang JC-S, Jeng S-F. Teleconsultation with the Mobile CameraPhone in Digital Soft-Tissue Injury: A Feasibility Study. Plast Reconstr Surg 2004; 114(7): 1776-1782.

7. Wiseman JT, Fernandes-Taylor S, Barnes ML, Tomsejova A, Saunders RS, Kent KC. Conceptualizing smartphone use in outpatient wound assessment: patients' and caregivers' willingness to use technology. J Surg Res 2015; 198(1): 245-251.

8. Roth AC, Reid JC, Puckett CL, Concannon MJ. Digital Images in the Diagnosis of Wound Healing Problems. Plast Reconstr Surg 1999; 103(2): 483-486.

9. Braun RP, Vecchietti JL, Thomas L, Prins C, French LE, Gewirtzman AJ, Saurat JH, Salomon D. Telemedical Wound Care Using a New Generation of Mobile Telephones. Arch Dermatol 2005; 141(2): 254-258.

10. Wirthlin DJ, Buradagunta S, Edwards RA, Brewster DC, Cambria RP, Gertler JP, LaMuraglia GM, Jordan De, Kvedar JC, Abbott WM. Telemedicine in vascular surgery $\bigotimes$ : Feasibility of digital imaging for remote management of wounds. J Vasc Surg 1998; 27(6): 1089-1100.

11.Landman A, Emani S, Carlile N, Rosenthal DI, Semakov S, Pallin DJ, Poon EG. A Mobile App for Securely Capturing and Transferring Clinical Images to the Electronic Health Record冈: Description and Preliminary Usability Study. JMIR mHealth uHealth 2015; 3(1): 1-8.

12.Dala-Ali BM, Lloyd MA, Al-Abed Y. The uses of the iPhone for surgeons. Surgeon Surg 2011; 9(1): 44-48.

13. Harris PA, Taylor R, Thielke R, Payne J, Gonzalez N, Conde JG. Research electronic data capture (REDCap) - a metadata-driven methodology and workflow process for providing translational research informatics support. J Biomed Inform 2009; 42(2): 377-381.

14. Beard HR, Hamid KS. Worth a thousand words: Integrating clinical photographs into an electronic medical record. Healthc 2014; 2(1): 22-25. 